\title{
Cerebral Microbleeds in Cats Undergoing Brain MRI: A Retrospective Study of Epidemiology and Clinical Associations
}

\author{
Marybeth Tan ${ }^{1}$, Katia Marioni-Henry ${ }^{1,2}$
}

\author{
${ }^{1}$ The Royal (Dick) School of Veterinary Studies, The University of Edinburgh ${ }^{2}$ The Roslin Institute
}

\section{Introduction}

Cerebral microbleeds (CMBs) are well-defined, round or ovoid, black or very hypointense signals on T2*-weighted MRI of the brain (Greenberg et al., 2009). In humans, CMBs are increasingly recognised as subclinical markers for haemorrhagic stroke and dementia and there is accumulating evidence that the spatial distribution of CMBs might be reflective of specific underlying vascular pathologies and may also have direct effects on neurological function. This study was conducted to determine the epidemiology of CMBs in cats undergoing brain MRI and if there is a correlation between the presence of CMBs and systemic disease.

\section{Materials and Method}

In this retrospective case-control study, magnetic resonance images/findings from all client-owned cats presented to the R(D)SVS Hospital for Small Animals that underwent brain MRI including a T2*weighted transverse series, regardless of the indication for MRI, from January 2010 to August 2020 were used to identify the number and distribution of cerebral microbleeds. The MR images were acquired using either a Philips Gyroscan Intera or a Siemens Magnetom Avanto 1.5 Tesla. Signalment, diagnostic and clinicopathological data (subject to availability/inclusion in records) were collected and assessed with the group of cats with cerebral microbleeds compared to age and sex/neuter status-matched controls. A Fisher exact test was used to examine the effects of neurological, systemic, and clinicopathological abnormalities on the presence of CMBs in matched case-control sets. A $P$-value of $<.05$ was considered statistically significant.

\section{Results}

Among the 107 cats that underwent brain MRI within the study period, 67 cats had a T2*-weighted transverse series. Out of the 67 cats, 14 cats (20.9\%) had CMBs and all had more than 1 CMB identified, with a median of 4.5 (range $2-11$ ). The majority of the cats $(10 / 14 ; 71.4 \%$ ) had CMBs in the cerebral cortex, $6 / 14(42.9 \%)$ cats had CMBs limited to the cerebral cortex and cerebellum, $2 / 14(14.3 \%)$ cats had CMBs in both the cerebral cortex, deep forebrain structures and brainstem and 9/14 $(64.2 \%)$ cats had CMBs in deep forebrain structures, brainstem and cerebellum.

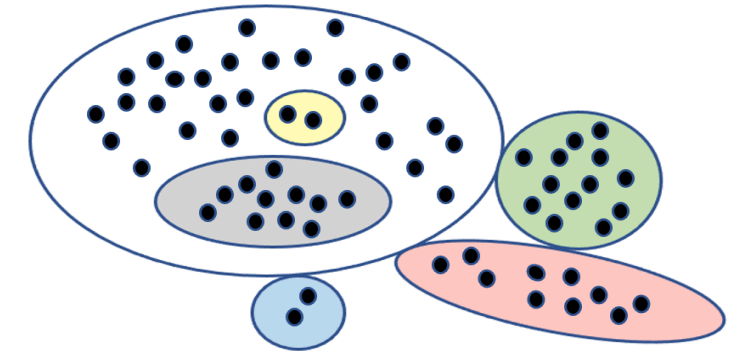

Figure 1 - Distribution of CMBs in the feline brain. Each black dot represents $1 \mathrm{CMB}$. White - Cerebral cortex, Yellow - Thalamus, Grey Basal nuclei, Green - Cerebellum, Pink - Brainstem, Blue - Pituitary gland

Cerebral microbleeds were more common in cats older than 12 years
$(P<0.05)$ and a diagnosis of renal disease was significantly associated $(P=0.01)$ with $\mathrm{CMBs}$

There was a lack of association between systolic blood pressure and the presence of CMBs.
Table 1 - Association of neurologic abnormalities/systemic diseases with presence of cerebral microbleeds (CMBs) in age- and sex/neuter status-matched cats undergoing 1.0or $1.5 \mathrm{~T}$ magnetic resonance imaging (MRI). For all variables, 14 cats with $\mathrm{CMBs}$ and 13 cats without CMBs were evaluated. No suitable match was found for case number 11, a 24year-old, neutered male, domestic short-haired cat.

\begin{tabular}{lccc}
\hline Neurologic dysfunction & With CMBs & Without CMB & $P$-value \\
\hline Seizures & $2 / 14$ & $5 / 13$ & 0.21 \\
Other intracranial signs & $9 / 14$ & $9 / 13$ & 1.0 \\
Vestibular dysfunction & $7 / 14$ & $2 / 13$ & 0.10 \\
Spinal cord/ neuromuscular disease & $5 / 14$ & $1 / 13$ & 0.17 \\
\hline Systemic diseases & With CMBs & Without CMB & $P$-value \\
\hline Renal disease & $7 / 14$ & $0 / 13$ & 0.01 \\
Systemic hypertension & $4 / 14$ & $1 / 13$ & 0.32 \\
Hyperthyroidism & $1 / 14$ & $5 / 13$ & 0.08 \\
Diabetes mellitus & $0 / 14$ & $0 / 13$ & NA \\
Other & $8 / 14$ & $5 / 13$ & 0.45 \\
No disease & $1 / 14$ & $4 / 13$ & 0.32
\end{tabular}
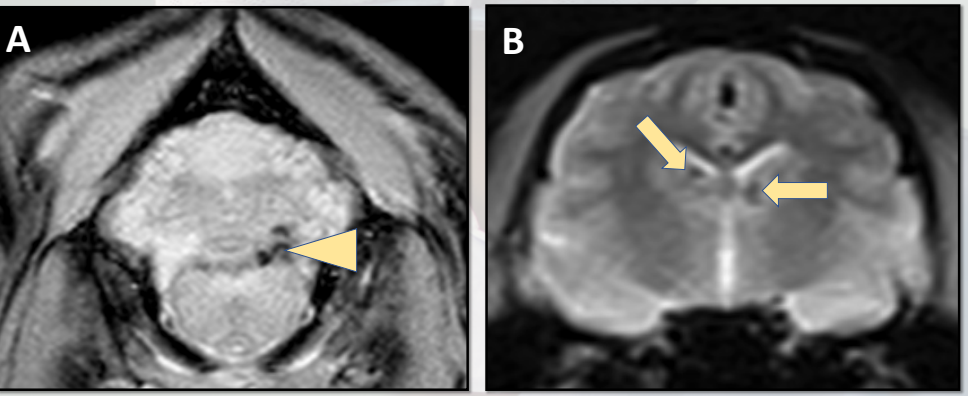

Figure 2 - Magnetic resonance images showing cerebral microbleeds (CMBs). (A) T2* weighted transverse image at the level of cerebellum and medulla oblongata showing multiple areas of $\mathrm{T}^{*}$ signal loss (see yellow arrowhead) associated with the choroid plexus of the $4^{\text {th }}$ ventricle of a 24 -year-old, neutered male cat with systemic hypertension and chronic kidney disease. (B) T2*-weighted transverse image of the forebrain at the level of the caudal nuclei showing multiple areas of T2* signal loss (see yellow arrows) each about $1.25 \mathrm{~mm}$ in diameter, in the caudate nucleus of a 14-year-old, neutered female cat with hyperadrenocorticism and renal disease.

\section{Limitations}

Due to the retrospective nature of this study, the limitations are the small sample size, lack of data regarding many variables of interest, and different MR systems used during the period of time analysed.

\section{Conclusion}

The prevalence of CMBs in cats in this study was $20.9 \%, 2.2$ times more than that reported in a recent similar study in dogs $9.3 \%$ (Kerwin et al., 2017), which may be due to the higher prevalence of chronic kidney disease in cats compared to dogs (Bartlett et al., 2010). Systemic hypertension may also be a major cause of CMBs in cats, as reflected by the distribution of CMBs in the deep forebrain structures, brainstem and cerebellum of cats, indeed, a similar distribution of CMBs in humans has been associated with hypertensive or atherosclerotic microangiopathy (Vernooij et al., 2008).

\section{References}

Bartlett, P. C., Van Buren, J. W., Neterer, M., \& Zhou, C. (2010). Disease surveillance and referral bias in the veterinary medical database. Preventive veterinary medicine, 94(3-4), 264-271.

Greenberg, S. M., Vernooij, M. W., et al. (2009) 'Cerebral microbleeds: a guide to detection and interpretation', The Lancet Neurology. NIH Public Access, pp. 165-174.

Kerwin, S.C., Levine, J.M., et al., (2017) Putative cerebral microbleeds in dogs undergoing magnetic resonance imaging of the head: a retrospective study of demographics, clinical associations, and relationship to case outcome. Journal of veterinary internal medicine, 31(4), pp.1140-1148.

Vernooij, M. W. et al. (2008) 'Prevalence and risk factors of cerebral microbleeds: The Rotterdam Scan Study', Neurology. Lippincott Williams and Wilkins, 70(14), pp. 1208-1214. 